\title{
What does the IMF really tell us about star formation?
}

\author{
M. B. N. Kouwenhoven ${ }^{1,2}$ and S. P. Goodwin ${ }^{2}$ \\ ${ }^{1}$ The Kavli Institute for Astronomy and Astrophysics, Peking University, Yi He Yuan Lu 5, \\ Hai Dian Qu Beijing 100871, P. R. China \\ email: kouwenhoven@kiaa.pku.edu.cn \\ ${ }^{2}$ University of Sheffield, Hicks Building, Hounsfield Road, Sheffield S3 7RH, United Kingdom \\ email: s.goodwin@sheffield.ac.uk
}

\begin{abstract}
Obtaining accurate measurements of the initial mass function (IMF) is often considered to be the key to understanding star formation, and a universal IMF is often assumed to imply a universal star formation process. Here, we illustrate that different modes of star formation can result in the same IMF, and that, in order to truly understand star formation, a deeper understanding of the primordial binary population is necessary. Detailed knowledge on the binary fraction, mass ratio distribution, and other binary parameters, as a function of mass, is a requirement for recovering the star formation process from stellar population measurements.
\end{abstract}

Keywords. binaries: general, stars: formation, mass function

Finding the IMF has been a major goal of stellar and galactic astrophysics, as it provides crucial information about the star formation process (e.g., Salpeter 1955; Kroupa 2002; Chabrier 2003; Bonnell, Larson \& Zinnecker 2007). The core mass function (CMF) was shown to have a shape very similar to the IMF (e.g., Motte et al. 2001; Simpson, Nutter \& Ward-Thompson 2008, and numerous others), suggesting a link between the two (see, in particular, Alves, Lombardi \& Lada 2007; Goodwin et al. 2008). However, this link is not trivial. It is known that most stars are part of a binary system (Lada \& Lada 2003), and that most (possibly even all) stars have formed in binary or multiple systems (e.g., Goodwin \& Kroupa 2005, Kouwenhoven et al. 2005, 2007). The IMF represents the mass distribution over all stars (including companion stars), and therefore depends on the properties of the primordial binaries (in particular the binary fraction and mass ratio distribution). The solution to the problem of recovering the outcome of star formation process is not unique, and the IMF therefore has to be interpreted with care.

To illustrate this issue, we generate stellar populations from a CMF, and compare the IMFs resulting from different formation processes (i.e., different binary fractions and mass ratio distributions). We assume a universal CMF similar the observed CMF of Alves et al. (2007) and Nutter \& Ward-Thompson (2007), which has an average mass of $\sim 1 M_{\odot}$, and a width of $\sigma_{\log M_{C}} \sim 0.5$. Each core is then converted into a system mass $M_{S}$, given a certain core-to-star formation efficiency (SFE). Finally, a fraction of these systems is split up into binaries with a certain mass ratio distribution $f(q)$, following the prescription for SCP-I described in Kouwenhoven et al. (2009).

Fig. 1 shows the IMFs (bottom panels) resulting from models with different mass ratio distributions (top panels), where we have set the binary fraction and SFE to unity. Despite the star formation process (here represented by the mass ratio distribution) being completely different, the IMFs are similar. In fact, the four IMFs are statistically identical for any observational sample with a realistic number of stars. The results can be generalised for populations with a mass-dependent binary fraction, $f(q)$, and SFE; for 

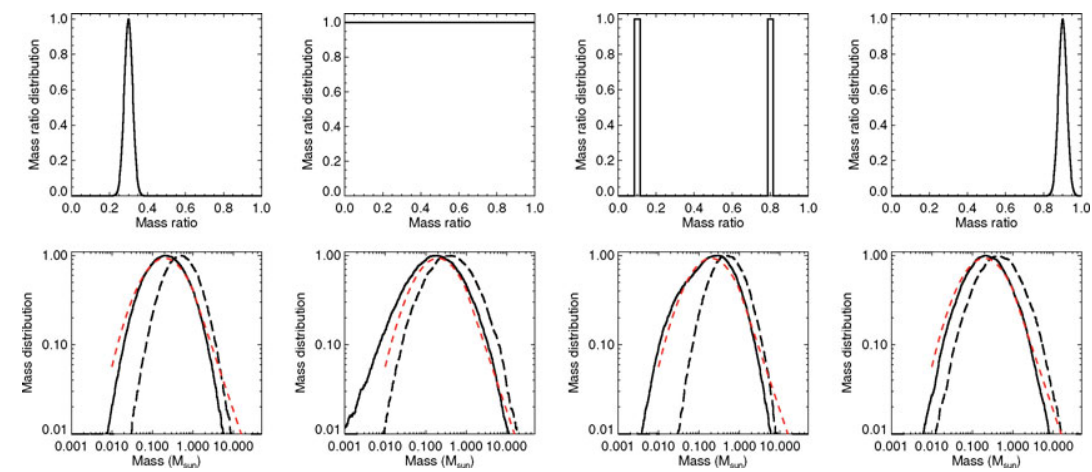

Figure 1. In the bottom panels we show the IMFs (solid black lines) formed from a given system mass function (SMF; dashed line) with an $f(q)$ as shown in the top panel, assuming a binary fraction of unity. The IMFs are compared to a canonical Chabrier (2003) IMF (light red dashed lines). The means and variances of the SMFs that give the best fits are (from left to right) $\mu_{\log 10 M}=-0.3,-0.4,-0.3$ and -0.35 , and $\sigma_{\log 10 M}=0.4,0.55,0.4$, and 0.5 , respectively.

details we refer to Goodwin \& Kouwenhoven (2009). With the examples shown in Fig. 1 we do not imply that all modes of star formation result in the same IMF, but merely that it is not possible to constrain star formation using the IMF alone.

It is often assumed that stellar populations with the same IMF must have had an identical formation process. Here, we illustrate that completely different modes of star formation may result in the same IMF, and that an observed IMF has to be interpreted with care. On the other hand, if two star clusters are found to have a different IMF, star formation must have been different. The IMF is an extremely important concept in understanding star formation, and theories and simulations of star formation should result in an IMF identical to the observed IMF. The general shape of the IMF is primarily determined by the properties of the CMF. However, to recover the details of star formation, the IMF alone is not enough; measuring the binary fraction, $f(q)$, and other binary parameters as a function of stellar mass is a prerequisite.

\section{Acknowledgements}

MBNK was supported by the Peter and Patricia Gruber Foundation through the PPGF fellowship, and by PPARC/STFC (grant number PP/D002036/1).

\section{References}

Alves, J., Lombardi, M., \& Lada, C. J. 2007, A\&A, 462, L17

Bonnell, I. A., Larson, R. B., \& Zinnecker, H. 2007, Protostars and Planets V, 149

Chabrier, G. 2003, PASP, 115, 763

Duquennoy, A. \& Mayor, M. 1991, A\&A, 248, 485

Goodwin, S. P., Nutter, D., Kroupa, P., Ward-Thompson, D., \& Whitworth, A. P. 2008, A\&A, 477,823

Goodwin, S. P. \& Kouwenhoven, M. B. N. 2009, MNRAS, 397, L36

Goodwin, S. P. \& Kroupa, P. 2005, A\&A, 439, 565

Kouwenhoven, M. B. N., Brown, A. G. A., Zinnecker, H., Kaper, L., \& Portegies Zwart, S. F. 2005, A\&A, 430, 137

Kouwenhoven, M. B. N., Brown, A. G. A., \& Kaper, L. 2007, A\&A, 464, 581

Kouwenhoven, M. B. N., Brown, A. G. A., Goodwin, S. P., Portegies Zwart, S. F., \& Kaper, L. 2009, A\&A, 493, 979

Kroupa, P. \& Boily, C. M. 2002, MNRAS, 336, 1188

Motte, F., André, P., Ward-Thompson, D., \& Bontemps, S. 2001, A\&A, 372, L41

Simpson, R. J., Nutter, D., \& Ward-Thompson, D. 2008, MNRAS, 391, 205 